\title{
A HALF-INVERSE PROBLEM FOR THE SINGULAR DIFFUSION OPERATOR WITH JUMP CONDITIONS
}

\author{
ABDULLAH ERGÜN
}

Received 02 June, 2020

\begin{abstract}
In this paper, half inverse spectral problem for diffusion operator with jump conditions dependent on the spectral parameter and discontinuity coefficient is considered. The half inverse problems is studied of determining the coefficient and two potential functions of the boundary value problem its spectrum by Hocstadt-Lieberman and Yang-Zettl methods. We show that two potential functions on the whole interval and the parameters in the boundary and jump conditions can be determined from the spectrum.
\end{abstract}

2010 Mathematics Subject Classification: 34K08; 34L05; 34K06; 34L10; 34E05

Keywords: differential equations, discontinuous function, singular diffusion operator

\section{INTRODUCTION AND PRELIMINARIES}

We consider the boundary value problem of the form

$$
l(y):=-y^{\prime \prime}+[2 \lambda p(x)+q(x)] y=\lambda^{2} \delta(x) y, x \in[0, \pi] /\left\{a_{1}, a_{2}\right\}
$$

with the boundary conditions

$$
y^{\prime}(0)=0, \quad y(\pi)=0
$$

and the jump conditions

$$
\begin{aligned}
y\left(a_{1}+0\right) & =\alpha_{1} y\left(a_{1}-0\right) \\
y^{\prime}\left(a_{1}+0\right) & =\beta_{1} y^{\prime}\left(a_{1}-0\right)+i \lambda \gamma_{1} y\left(a_{1}-0\right) \\
y\left(a_{2}+0\right) & =\alpha_{2} y\left(a_{2}-0\right) \\
y^{\prime}\left(a_{2}+0\right) & =\beta_{2} y^{\prime}\left(a_{2}-0\right)+i \lambda \gamma_{2} y\left(a_{2}-0\right),
\end{aligned}
$$

where $\lambda$ is a spectral parameter, $p(x) \in W_{2}^{1}[0, \pi], q(x) \in L_{2}[0, \pi]$ are real valued functions, $a_{1} \in\left[0, \frac{\pi}{2}\right], a_{2} \in\left[\frac{\pi}{2}, \pi\right], \alpha_{1}, \alpha_{2}, \gamma_{1}, \gamma_{2}$ are real numbers, $\left|\alpha_{i}-1\right|^{2}+\gamma_{i}^{2} \neq$ $0\left(\alpha_{i}>0 ; i=1,2\right), \beta_{i}=\frac{1}{\alpha_{i}}(i=1,2)$ and

$$
\delta(x)=\left\{\begin{array}{l}
\alpha^{2}, x \in\left(0, \frac{\pi}{2}\right) \\
\beta^{2}, x \in\left(\frac{\pi}{2}, \pi\right)
\end{array}\right.
$$


for $0<\alpha<\beta<1, \alpha+\beta>1$.

The inverse problems consist in recovering the coefficients of an operator from their spectral characteristics. A lot of study were done the inverse spectral problem for Sturm-Liouville operators and diffusion operators [1,2,4-26]. The first results on inverse problems theory of Sturm-Liouville operators were given by Ambarzumyan [3]. The half inverse problems for Sturm-Liouville equations; the known potential in half interval is determined by the help of a one spectrum over the interval. New results on the half inverse problem were obtained by Hochstadt and Lieberman [11]. They proved the spectrum of the problem as:

$$
\begin{aligned}
-y^{\prime \prime}+q(x) y & =\lambda y, x \in[0,1] \\
y^{\prime}(0)-h y(0) & =0 \\
y^{\prime}(1)+H y(1) & =0
\end{aligned}
$$

and potential $q(x)$ on the $\left(\frac{1}{2}, 1\right)$ uniquely determine the potential $q(x)$ on the whole interval $[0,1]$ almost everywhere. Hald [10] proved similar results in the case when there exists a impulsive conditions inside the interval. Many studies have been done by different authors for half inverse problems using these methods [14,19]. In [19] the authors studied the existence of the solution for the half-inverse problem of SturmLiouville problems and gave method of reconstructing this solution under same conditions by Sakhnovich [19]. Recently, some new uniqueness results have been given on the inverse or half inverse spectral analysis of differential operators. Koyunbakan and Panakhov [14] proved the half inverse problem for diffusion operator on the finite interval $[0, \pi]$. Ran Zhang, Xiao-Chuan Xu, Chuan-Fu Yang and Natalia Pavlovna Bondarenko proved the determination of the impulsive Sturm-Liouville operator from a set of eigenvalues [26]. The purpose of this study is to prove half inverse problem by using the Hocstadt- Lieberman and Yang-Zettl methods for the following equations

$$
\begin{gathered}
\tilde{l}(y):=-y^{\prime \prime}+[2 \lambda \tilde{p}(x)+\tilde{q}(x)] y=\lambda^{2} \tilde{\delta}(x) y, x \in[0, \pi] /\left\{a_{1}, a_{2}\right\} \\
y^{\prime}(0)=0, y(\pi)=0 \\
y\left(a_{1}+0\right)=\tilde{\alpha}_{1} y\left(a_{1}-0\right) \\
y^{\prime}\left(a_{1}+0\right)=\tilde{\beta}_{1} y^{\prime}\left(a_{1}-0\right)+i \lambda \tilde{\gamma}_{1} y\left(a_{1}-0\right) \\
y\left(a_{2}+0\right)=\tilde{\alpha}_{2} y\left(a_{2}-0\right) \\
y^{\prime}\left(a_{2}+0\right)=\tilde{\beta}_{2} y^{\prime}\left(a_{2}-0\right)+i \lambda \tilde{\gamma}_{2} y\left(a_{2}-0\right) .
\end{gathered}
$$

Lemma 1. Let $p(x) \in W_{2}^{1}(0, \pi), q(x) \in L_{2}(0, \pi), M(x, t), N(x, t)$ are summable functions on $[0, \pi]$ such that the representation for each $x \in[0, \pi] /\left\{a_{1}, a_{2}\right\} . \varphi(x, \lambda)$ 
is the solution of the equations (1.1), providing boundary conditions (1.2) and discontinuity conditions (1.3)-(1.6),

$$
\varphi(x, \lambda)=\varphi_{0}(x, \lambda)+\int_{0}^{x} M(x, t) \cos \lambda t d t+\int_{0}^{x} N(x, t) \sin \lambda t d t
$$

for $0<x<\frac{\pi}{2}$, that is given as

$$
\begin{aligned}
\varphi_{0}(x, \lambda)= & \left(\beta_{1}^{+}+\frac{\gamma_{1}}{2 \alpha}\right) \cos \left[\lambda \xi^{+}(x)-\frac{1}{\alpha} \int_{a_{1}}^{x} p(t) d t\right] \\
& +\left(\beta_{1}^{-}-\frac{\gamma_{1}}{2 \alpha}\right) \cos \left[\lambda \xi^{-}(x)+\frac{1}{\alpha} \int_{a_{1}}^{x} p(t) d t\right],
\end{aligned}
$$

for $\frac{\pi}{2}<x \leq \pi$,

$$
\begin{aligned}
\varphi_{0}(x, \lambda)= & \left(\beta_{2}^{+}+\frac{\gamma_{2}}{2 \beta}\right) \cos \left[\lambda k^{+}(\pi)-\frac{1}{\beta} \int_{a_{2}}^{\pi} p(t) d t\right] \\
& +\left(\beta_{2}^{-}+\frac{\gamma_{2}}{2 \beta}\right) \cos \left[\lambda k^{-}(\pi)-\frac{1}{\beta} \int_{a_{2}}^{\pi} p(t) d t\right] \\
& +\left(\beta_{2}^{-}-\frac{\gamma_{2}}{2 \beta}\right) \cos \left[\lambda s^{+}(\pi)+\frac{1}{\beta} \int_{a_{2}}^{\pi} p(t) d t\right] \\
& +\left(\beta_{2}^{+}-\frac{\gamma_{2}}{2 \beta}\right) \cos \left[\lambda s^{-}(\pi)+\frac{1}{\beta} \int_{a_{2}}^{\pi} p(t) d t\right],
\end{aligned}
$$

where $\xi^{ \pm}(x)= \pm \alpha x \mp \alpha a_{1}+a_{1}, \quad k^{ \pm}(x)=\xi^{+}\left(a_{2}\right) \pm \beta x \mp \beta a_{2}$,

$$
s^{ \pm}(x)=\xi^{-}\left(a_{2}\right) \pm \beta x \mp \beta a_{2}, \quad \beta_{1}^{\mp}=\frac{1}{2}\left(\alpha_{1} \mp \frac{\beta_{1}}{\alpha}\right), \quad \beta_{2}^{\mp}=\frac{1}{2}\left(\alpha_{2} \mp \frac{\alpha \beta_{2}}{\beta}\right) .
$$

Thus, the following relations hold:

$$
\begin{aligned}
& \text { If } p(x) \in W_{2}^{2}(0, \pi), q(x) \in W_{2}^{1}(0, \pi) \\
& \qquad \begin{array}{r}
\frac{\partial^{2} M(x, t)}{\partial x^{2}}-\rho(x) \frac{\partial^{2} M(x, t)}{\partial t^{2}}=2 p(x) \frac{\partial N(x, t)}{\partial t}+q(x) M(x, t) \\
\frac{\partial^{2} N(x, t)}{\partial x^{2}}-\rho(x) \frac{\partial^{2} N(x, t)}{\partial t^{2}}=-2 p(x) \frac{\partial M(x, t)}{\partial t}+q(x) N(x, t)
\end{array} \\
& \quad M\left(x, \varsigma^{+}(x)\right) \cos \frac{\beta(x)}{\alpha}+N\left(x, \varsigma^{+}(x)\right) \sin \frac{\beta(x)}{\alpha}=\left(\beta_{1}^{+}+\frac{\gamma_{1}}{2 \alpha}\right) \int_{0}^{x}\left(q(t)+\frac{p^{2}(t)}{\alpha^{2}}\right) d t \\
& \quad M\left(x, \varsigma^{+}(x)\right) \sin \frac{\beta(x)}{\alpha}-N\left(x, \varsigma^{+}(x)\right) \cos \frac{\beta(x)}{\alpha}=\left(\beta_{1}^{+}+\frac{\gamma_{1}}{2 \alpha}\right)(p(x)-p(0)) \\
& M\left(x, k^{+}(x)+0\right)-M\left(x, k^{+}(x)-0\right) \\
& =-\left(\beta_{2}^{+}+\frac{\gamma_{2}}{2 \beta}\right)(p(x)-p(0)) \sin \frac{\omega(x)}{\beta}-\left(\beta_{2}^{+}+\frac{\gamma_{2}}{2 \beta}\right) \int_{0}^{x}\left(q(t)+\frac{p^{2}(t)}{\beta^{2}}\right) d t \cos \frac{\omega(x)}{\beta} \\
& N\left(x, k^{+}(x)+0\right)-N\left(x, k^{+}(x)-0\right)
\end{aligned}
$$




$$
\begin{aligned}
& =\left(\beta_{2}^{+}+\frac{\gamma_{2}}{2 \beta}\right)(p(x)-p(0)) \cos \frac{\omega(x)}{\beta}-\left(\beta_{2}^{+}+\frac{\gamma_{2}}{2 \beta}\right) \int_{0}^{x}\left(q(t)+\frac{p^{2}(t)}{\beta^{2}}\right) d t \sin \frac{\omega(x)}{\beta}, \\
& \left.\frac{\partial M(x, t)}{\partial t}\right|_{t=0}=N(x, 0)=0, \\
& \text { where } \beta(x)=\int_{0}^{x} p(t) d t, \omega(x)=\int_{a_{2}}^{x} p(t) d t+\int_{0}^{a_{1}} p(t) d t .
\end{aligned}
$$

The proof is done as in [6].

Definition 1. The function $\Delta(\lambda)$ is called the characteristic function of the eigenvalues $\left\{\lambda_{n}\right\}$ of the problem (1.1)-(1.6). $\tilde{\Delta}(\lambda)$ is called the characteristic function of the eigenvalues $\left\{\tilde{\lambda}_{n}\right\}$ of the problem (1.7)-(1.12).

Let $\lambda=s^{2}, s=\sigma+i \tau, \sigma, \tau \in \mathrm{R}$. The solution $\varphi(x, \lambda)$ of (1.1)-(1.6) has the following asymptotic formulas hold on for $|\lambda| \rightarrow \infty$, for $0<x<\frac{\pi}{2}$,

$$
\varphi(x, \lambda)=\frac{1}{2}\left(\frac{\alpha_{1}}{2} \mp \frac{\beta_{1}}{2 \alpha}+\frac{\gamma_{1}}{2 \alpha}\right) \exp \left(-i\left(\lambda \xi^{+}(x)-\frac{v(x)}{\alpha}\right)\right)\left(1+O\left(\frac{1}{\lambda}\right)\right),
$$

for $\frac{\pi}{2}<x \leq \pi$,

$$
\varphi(x, \lambda)=\frac{1}{2}\left(\frac{\alpha_{2}}{2}+\frac{\alpha \beta_{2}}{2 \beta}+\frac{\gamma_{2}}{2 \beta}\right) \exp \left(-i\left(\lambda k^{+}(x)-\frac{t(x)}{\beta}\right)\right)\left(1+O\left(\frac{1}{\lambda}\right)\right),
$$

where $v(x)=\int_{a_{1}}^{x} p(t) d t, t(x)=\int_{a_{2}}^{x} p(t) d t$.

In this study, if $q(x)$ and $p(x)$ to be known almost everywhere on $\left(\frac{\pi}{2}, \pi\right)$, it is sufficient to determine uniquely $p(x)$ and $q(x)$ on the whole interval $(0, \pi)$.

\section{MAIN RESULT}

If $\varphi_{0}(x, \lambda)$ is a nontrivial solution of equation (1.1) with conditions (1.2)-(1.6), then $\lambda_{0}$ is called an eigenvalue. Additionally, $\varphi_{0}(x, \lambda)$ is called the eigenfunction of the problem corresponding to the eigenvalue $\lambda_{0}$. $\left\{\lambda_{n}\right\}$ are the eigenvalues of the problem.

Lemma 2. If $\lambda_{n}=\tilde{\lambda}_{n}, \frac{\alpha}{\tilde{\alpha}}=\frac{\beta}{\tilde{\beta}}$ then $\alpha=\tilde{\alpha}$ and $\beta=\tilde{\beta}$ for all $n \in \mathrm{N}$.

Proof. Since $\lambda_{n}=\tilde{\lambda}_{n}$ and $\Delta(\lambda), \tilde{\Delta}(\lambda)$ are entire functions in $\lambda$ of order one by Hadamard factorization theorem for $\lambda \in \mathrm{C}$

$$
\Delta(\lambda) \equiv C \tilde{\Delta}(\lambda) .
$$

On the other hand, (1.1) can be written as

$$
\Delta_{0}(\lambda)-C \tilde{\Delta}_{0}(\lambda)=C\left[\tilde{\Delta}(\lambda)-\tilde{\Delta}_{0}(\lambda)\right]-\left[\Delta(\lambda)-\Delta_{0}(\lambda)\right] .
$$

Hence

$$
C\left[\tilde{\Delta}(\lambda)-\tilde{\Delta}_{0}(\lambda)\right]-\left[\Delta(\lambda)-\Delta_{0}(\lambda)\right]
$$




$$
\begin{aligned}
= & \left(\beta_{2}^{+}+\frac{\gamma_{2}}{2 \beta}\right) \cos \left[\lambda k^{+}(\pi)-\frac{w(\pi)}{\beta}\right]+\left(\beta_{2}^{-}+\frac{\gamma_{2}}{2 \beta}\right) \cos \left[\lambda k^{-}(\pi)-\frac{w(\pi)}{\beta}\right] \\
& +\left(\beta_{2}^{-}-\frac{\gamma_{2}}{2 \beta}\right) \cos \left[\lambda s^{+}(\pi)+\frac{w(\pi)}{\beta}\right]+\left(\beta_{2}^{+}-\frac{\gamma_{2}}{2 \beta}\right) \cos \left[\lambda s^{-}(\pi)+\frac{w(\pi)}{\beta}\right] \\
& -C\left(\tilde{\beta}_{2}^{+}+\frac{\tilde{\gamma}_{2}}{2 \tilde{\beta}}\right) \cos \left[\lambda k^{+}(\pi)-\frac{\tilde{w}(\pi)}{\tilde{\beta}}\right]-C\left(\tilde{\beta}_{2}^{-}+\frac{\tilde{\gamma}_{2}}{2 \tilde{\beta}}\right) \cos \left[\lambda k^{-}(\pi)-\frac{\tilde{w}(\pi)}{\tilde{\beta}}\right] \\
& -C\left(\tilde{\beta}_{2}^{-}-\frac{\tilde{\gamma_{2}}}{2 \tilde{\beta}}\right) \cos \left[\lambda s^{+}(\pi)+\frac{\tilde{w}(\pi)}{\tilde{\beta}}\right]-C\left(\tilde{\beta}_{2}^{+}-\frac{\tilde{\gamma}_{2}}{2 \tilde{\beta}}\right) \cos \left[\lambda s^{-}(\pi)+\frac{\tilde{w}(\pi)}{\beta}\right] .
\end{aligned}
$$

If we multiply both sides of (2.1) by $\cos \left[\lambda k^{+}(\pi)-\frac{w(\pi)}{\beta}\right]$ and integrate with respect to $\lambda$ in $(\varepsilon, T)$, ( $\varepsilon$ is a sufficiently small positive number) for any positive real number $T$, then we get

$$
\begin{aligned}
& \int_{\varepsilon}^{T}\left(C\left[\tilde{\Delta}(\lambda)-\tilde{\Delta}_{0}(\lambda)\right]-\left[\Delta(\lambda)-\Delta_{0}(\lambda)\right]\right) \cos \left[\lambda k^{+}(\pi)-\frac{w(\pi)}{\beta}\right] d \lambda \\
& =\int_{\varepsilon}^{T}\left\{\left(\beta_{2}^{+}+\frac{\gamma_{2}}{2 \beta}\right) \cos \left[\lambda k^{+}(\pi)-\frac{w(\pi)}{\beta}\right]+\left(\beta_{2}^{-}+\frac{\gamma_{2}}{2 \beta}\right) \cos \left[\lambda k^{-}(\pi)-\frac{w(\pi)}{\beta}\right]\right. \\
& \quad+\left(\beta_{2}^{-}-\frac{\gamma_{2}}{2 \beta}\right) \cos \left[\lambda s^{+}(\pi)+\frac{w(\pi)}{\beta}\right]+\left(\beta_{2}^{+}-\frac{\gamma_{2}}{2 \beta}\right) \cos \left[\lambda s^{-}(\pi)+\frac{w(\pi)}{\beta}\right] \\
& -C\left(\tilde{\beta}_{2}^{+}+\frac{\tilde{\gamma}_{2}}{2 \tilde{\beta}}\right) \cos \left[\lambda k^{+}(\pi)-\frac{\tilde{w}(\pi)}{\tilde{\beta}}\right]-C\left(\tilde{\beta}_{2}^{-}+\frac{\tilde{\gamma}_{2}}{2 \tilde{\beta}}\right) \cos \left[\lambda k^{-}(\pi)-\frac{\tilde{w}(\pi)}{\tilde{\beta}}\right] \\
& -C\left(\tilde{\beta}_{2}^{-}-\frac{\tilde{\gamma}_{2}}{2 \tilde{\beta}}\right) \cos \left[\lambda s^{+}(\pi)+\frac{\tilde{w}(\pi)}{\tilde{\beta}}\right]-C\left(\tilde{\beta}_{2}^{+}-\frac{\tilde{\gamma}_{2}}{2 \tilde{\beta}}\right) \cos \left[\lambda s^{-}(\pi)+\frac{\tilde{w}(\pi)}{\beta}\right] d \lambda .
\end{aligned}
$$

And so

$$
\begin{aligned}
& \int_{\varepsilon}^{T}\left(C\left[\tilde{\Delta}(\lambda)-\tilde{\Delta}_{0}(\lambda)\right]-\left[\Delta(\lambda)-\Delta_{0}(\lambda)\right]\right) \cos \left[\lambda k^{+}(\pi)-\frac{w(\pi)}{\beta}\right] d \lambda \\
& =\int_{\varepsilon}^{T}\left(\beta_{2}^{+}+\frac{\gamma_{2}}{2 \beta}\right) \cos ^{2}\left[\lambda k^{+}(\pi)-\frac{w(\pi)}{\beta}\right] d \lambda \\
& \quad-C \int_{\varepsilon}^{T}\left(\tilde{\beta}_{2}^{+}+\frac{\tilde{\gamma}_{2}}{2 \tilde{\beta}}\right) \cos \left[\lambda k^{+}(\pi)-\frac{w(\pi)}{\beta}\right] \cos \left[\lambda k^{+}(\pi)-\frac{\tilde{w}(\pi)}{\tilde{\beta}}\right] d \lambda \\
& =\int_{\varepsilon}^{T} \frac{1}{2}\left(\beta_{2}^{+}+\frac{\gamma_{2}}{2 \beta}\right)+\frac{1}{2}\left(\beta_{2}^{+}+\frac{\gamma_{2}}{2 \beta}\right) \cos \left[2 \lambda k^{+}(\pi)-\frac{2 w(\pi)}{\beta}\right] d \lambda \\
& -C \int_{\varepsilon}^{T} \frac{1}{2}\left(\tilde{\beta}_{2}^{+}+\frac{\tilde{\gamma}_{2}}{2 \tilde{\beta}}\right)\left(\cos \left[2 \lambda k^{+}(\pi)-\frac{\tilde{w}(\pi)+w(\pi)}{\beta}\right]+\cos \left[\frac{w(\pi)-\tilde{w}(\pi)}{\tilde{\beta}}\right]\right) d \lambda,
\end{aligned}
$$


$\Delta(\lambda)-\Delta_{0}(\lambda)=O\left(\frac{1}{|\lambda|} e^{|I m \lambda| k^{+}(\pi)}\right), \tilde{\Delta}(\lambda)-\tilde{\Delta}_{0}(\lambda)=O\left(\frac{1}{|\lambda|} e^{|I m \lambda| k^{+}(\pi)}\right)$ for all $\lambda$ in $(\varepsilon, T)$

$$
\frac{C}{2}\left(\tilde{\beta}_{2}^{+}+\frac{\tilde{\gamma}_{2}}{2 \tilde{\beta}}\right)-\frac{1}{2}\left(\beta_{2}^{+}+\frac{\gamma_{2}}{2 \beta}\right)=O\left(\frac{1}{T}\right) .
$$

By letting $T$ tend to infinity we see that

$$
C=\frac{\tilde{\beta}_{2}^{+}+\frac{\tilde{\gamma}_{2}}{2 \tilde{\beta}}}{\beta_{2}^{+}+\frac{\gamma_{2}}{2 \beta}} .
$$

Similarly, if we multiply both side of $(2.1) \cos \left[\lambda k^{-}(\pi)-\frac{w(\pi)}{\beta}\right]$ and integrate again with respect to $\lambda$ in $(\varepsilon, T)$ and by letting $T$ tend to infinity, then we get

$$
C=\frac{\tilde{\beta}_{2}^{-}+\frac{\tilde{\gamma}_{2}}{2 \tilde{\beta}}}{\beta_{2}^{-}+\frac{\gamma_{2}}{2 \beta}} .
$$

But since $\alpha, \beta$ and $\tilde{\alpha}, \tilde{\beta}$ are positive, and $w^{+}(\pi)-\tilde{w}^{+}(\pi)=w^{-}(\pi)-\tilde{w}^{-}(\pi)$ we conclude that $C=1$. Hence $\frac{\tilde{\beta}_{2}^{+}}{\beta_{2}^{+}}=\frac{\tilde{\beta}_{2}^{-}}{\beta_{2}^{-}}$is obtained. We have therefore proved since $\alpha=\tilde{\alpha}$ that $\beta=\tilde{\beta}$.

The proof is completed.

Lemma 3. If $\lambda_{n}=\tilde{\lambda}_{n}$ then $\alpha_{i}=\tilde{\alpha}_{i}$ and $\gamma_{i}=\tilde{\gamma}_{i}(i=1,2)$ for all $n \in \mathrm{N}$.

The proof is done as in [6].

Theorem 1. Let $\left\{\lambda_{n}\right\}$ be the eigenvalues of both problem (1.1)-(1.6) and (1.7)(1.12). If $p(x)=\tilde{p}(x)$ and $q(x)=\tilde{q}(x)$ on $\left[\frac{\pi}{2}, \pi\right]$, then $p(x)=\tilde{p}(x)$ and $q(x)=\tilde{q}(x)$ are almost everywhere on $[0, \pi]$.

Proof of Theorem 1. Let function $\varphi(x, \lambda)$ be the solution of equation (1.1) under the conditions (1.2)-(1.6) and the function $\tilde{\varphi}(x, \lambda)$ the solution of equation (1.7) under the conditions (1.8)-(1.12) on $\left[0, \frac{\pi}{2}\right]$. The integral forms of the functions $\varphi(x, \lambda)$ and $\tilde{\varphi}(x, \lambda)$ can be obtained as follows

$$
\begin{aligned}
\varphi(x, \lambda)= & \left(\beta_{1}^{+}+\frac{\gamma_{1}}{2 \alpha}\right) \cos \left[\lambda \xi^{+}(x)-\frac{1}{\alpha} \int_{a_{1}}^{x} p(t) d t\right] \\
& +\left(\beta_{1}^{-}-\frac{\gamma_{1}}{2 \alpha}\right) \cos \left[\lambda \xi^{-}(x)+\frac{1}{\alpha} \int_{a_{1}}^{x} p(t) d t\right] \\
& +\int_{0}^{x} M(x, t) \cos \lambda t d t+\int_{0}^{x} N(x, t) \sin \lambda t d t
\end{aligned}
$$

and

$$
\tilde{\varphi}(x, \lambda)=\left(\tilde{\beta}_{1}^{+}+\frac{\tilde{\gamma}_{1}}{2 \alpha}\right) \cos \left[\lambda \xi^{+}(x)-\frac{1}{\alpha} \int_{a_{1}}^{x} \tilde{p}(t) d t\right]
$$




$$
\begin{aligned}
& +\left(\tilde{\beta}_{1}^{-}-\frac{\tilde{\gamma}_{1}}{2 \alpha}\right) \cos \left[\lambda \xi^{-}(x)+\frac{1}{\alpha} \int_{a_{1}}^{x} \tilde{p}(t) d t\right] \\
& +\int_{0}^{x} \tilde{M}(x, t) \cos \lambda t d t+\int_{0}^{x} \tilde{N}(x, t) \sin \lambda t d t .
\end{aligned}
$$

If we multiply equations (2.2) and (2.3):

$$
\begin{aligned}
& \varphi(x, \lambda) \cdot \tilde{\varphi}(x, \lambda)=\frac{S^{+} \tilde{S}^{+}}{2}\left[\cos \left(2 \lambda \xi^{+}(x)-K(x)\right)+\cos L(x)\right] \\
& +\frac{S^{+} \tilde{S}^{-}}{2}\left[\cos \left(2 \lambda a_{1} t-L(x)\right)+\cos \left(2 \lambda \alpha\left(x-a_{1}\right)-K(x)\right)\right] \\
& +\frac{S^{-} \tilde{S}^{+}}{2}\left[\cos \left(2 \lambda a_{1}+L(x)\right)+\cos \left(2 \lambda \alpha\left(x-a_{1}\right)+K(x)\right)\right] \\
& +\frac{S^{-} \tilde{S}^{-}}{2}\left[\cos \left(2 \lambda \xi^{-}(x)+L(x)\right)+\cos K(x)\right] \\
& +S^{+} \int_{0}^{x} \tilde{M}(x, t) \cos \left[\lambda \xi^{+}(x)-\frac{t(x)}{\alpha}\right] \cos \lambda t d t \\
& +S^{+} \int_{0}^{x} \tilde{N}(x, t) \cos \left[\lambda \xi^{+}(x)-\frac{t(x)}{\alpha}\right] \sin \lambda t d t \\
& +S^{-} \int_{0}^{x} \tilde{M}(x, t) \cos \left[\lambda \xi^{-}(x)+\frac{t(x)}{\alpha}\right] \cos \lambda t d t \\
& +S^{-} \int_{0}^{x} \tilde{N}(x, t) \cos \left[\lambda \xi^{-}(x)+\frac{t(x)}{\alpha}\right] \sin \lambda t d t \\
& +\tilde{S}^{+} \int_{0}^{x} M(x, t) \cos \left[\lambda \xi^{+}(x)-\frac{\tilde{t}(x)}{\alpha}\right] \cos \lambda t d t \\
& +\tilde{S}^{+} \int_{0}^{x} N(x, t) \cos \left[\lambda \xi^{+}(x)-\frac{\tilde{t}(x)}{\alpha}\right] \sin \lambda t d t \\
& +\tilde{S}^{-} \int_{0}^{x} M(x, t) \cos \left[\lambda \xi^{-}(x)+\frac{\tilde{t}(x)}{\alpha}\right] \cos \lambda t d t \\
& +\tilde{S}^{-} \int_{0}^{x} N(x, t) \cos \left[\lambda \xi^{-}(x)+\frac{\tilde{t}(x)}{\alpha}\right] \sin \lambda t d t \\
& +\left(\int_{0}^{x} M(x, t) \cos \lambda t d t\right)\left(\int_{0}^{x} \tilde{M}(x, t) \cos \lambda t d t\right) \\
& +\left(\int_{0}^{x} N(x, t) \sin \lambda t d t\right)\left(\int_{0}^{x} \tilde{N}(x, t) \sin \lambda t d t\right) \\
& +\left(\int_{0}^{x} M(x, t) \cos \lambda t d t\right)\left(\int_{0}^{x} \tilde{N}(x, t) \sin \lambda t d t\right)
\end{aligned}
$$




$$
+\left(\int_{0}^{x} \tilde{M}(x, t) \cos \lambda t d t\right)\left(\int_{0}^{x} N(x, t) \sin \lambda t d t\right),
$$

$$
\begin{aligned}
\varphi(x, \lambda) \cdot \tilde{\varphi}(x, \lambda)= & \frac{S^{+} \tilde{S}^{+}}{2}\left[\cos \left(2 \lambda \xi^{+}(x)-K(x)\right)+\cos L(x)\right] \\
& +\frac{S^{+} \tilde{S}^{-}}{2}\left[\cos \left(2 \lambda a_{1} t-L(x)\right)+\cos \left(2 \lambda \alpha\left(x-a_{1}\right)-K(x)\right)\right] \\
& +\frac{S^{-} \tilde{S}^{+}}{2}\left[\cos \left(2 \lambda a_{1}+L(x)\right)+\cos \left(2 \lambda \alpha\left(x-a_{1}\right)+K(x)\right)\right] \\
& +\frac{S^{-} \tilde{S}^{-}}{2}\left[\cos \left(2 \lambda \xi^{-}(x)+L(x)\right)+\cos K(x)\right] \\
& +\frac{1}{2}\left\{\int_{0}^{x} U_{c}(x, t) \cos (2 \lambda t-K(t)) d t-\int_{0}^{x} U_{s}(x, t) \sin (2 \lambda t-K(t)) d t\right\}
\end{aligned}
$$

is obtained, being

$$
\begin{aligned}
S^{ \pm} & =\left(\beta_{1}^{ \pm} \mp \frac{\gamma_{1}}{2 \alpha}\right), \tilde{S}^{ \pm}=\left(\tilde{\beta}_{1}^{ \pm} \mp \frac{\tilde{\gamma}_{1}}{2 \alpha}\right), K(x)=\frac{t(x)+\tilde{t}(x)}{2}, L(x)=\frac{t(x)-\tilde{t}(x)}{2} \\
U_{c}(x, t) & \\
= & S^{+} \tilde{M}\left(x, \xi^{+}(x)-2 t\right) \cos \left(K(t)-\frac{t(x)}{\alpha}\right)+S^{-} \tilde{M}\left(x, \xi^{-}(x)-2 t\right) \cos \left(K(t)-\frac{t(x)}{\alpha}\right) \\
& +\tilde{S}^{+} M\left(x, \xi^{+}(x)-2 t\right) \cos \left(K(t)-\frac{\tilde{t}(x)}{\alpha}\right)+\tilde{S}^{-} M\left(x, \xi^{-}(x)-2 t\right) \sin \left(K(t)-\frac{\tilde{t}(x)}{\alpha}\right) \\
& -S^{-} \tilde{N}\left(x, \xi^{+}(x)-2 t\right) \sin \left(K(t)-\frac{t(x)}{\alpha}\right)-S^{-} \tilde{N}\left(x, \xi^{-}(x)-2 t\right) \sin \left(K(t)-\frac{t(x)}{\alpha}\right) \\
& -\tilde{S}^{+} N\left(x, \xi^{+}(x)-2 t\right) \sin \left(K(t)-\frac{\tilde{t}(x)}{\alpha}\right)-\tilde{S}^{-} N\left(x, \xi^{-}(x)-2 t\right) \sin \left(K(t)-\frac{\tilde{t}(x)}{\alpha}\right) \\
& +K_{1}(x, t) \cos K(t)+K_{2}(x, t) \cos K(t)+M_{1}(x, t) \sin K(t)+M_{2}(x, t) \sin K(t), \\
U_{S} & (x, t) \\
= & S^{+} \tilde{M}\left(x, \xi^{+}(x)-2 t\right) \sin \left(K(t)-\frac{t(x)}{\alpha}\right)+S^{-} \tilde{M}\left(x, \xi^{-}(x)-2 t\right) \sin \left(K(t)-\frac{t(x)}{\alpha}\right) \\
& +\tilde{S}^{+} M\left(x, \xi^{+}(x)-2 t\right) \sin \left(K(t)-\frac{\tilde{t}(x)}{\alpha}\right)+\tilde{S}^{-} M\left(x, \xi^{-}(x)-2 t\right) \sin \left(K(t)-\frac{\tilde{t}(x)}{\alpha}\right) \\
& +S^{+} \tilde{N}\left(x, \xi^{+}(x)-2 t\right) \cos \left(K(t)-\frac{t(x)}{\alpha}\right)+S^{-} \tilde{N}\left(x, \xi^{-}(x)-2 t\right) \cos \left(K(t)-\frac{t(x)}{\alpha}\right) \\
& +\tilde{S}^{+} N\left(x, \xi^{+}(x)-2 t\right) \cos \left(K(t)-\frac{\tilde{t}(x)}{\alpha}\right)+\tilde{S}^{-} N\left(x, \xi^{-}(x)-2 t\right) \cos \left(K(t)-\frac{\tilde{t}(x)}{\alpha}\right) \\
& \left.+K_{1}(x, t) \sin K(t)+K_{2}(x, t) \sin K(t)\right),
\end{aligned}
$$




$$
\begin{aligned}
K_{1}(x, t) & =\int_{-x}^{x-2 t} M(x, s) \tilde{M}(x, s+2 t) d s+\int_{2 t-x}^{x} M(x, s) \tilde{M}(x, s+2 t) d s \\
K_{2}(x, t) & =\int_{-x}^{x-2 t} N(x, s) \tilde{N}(x, s+2 t) d s+\int_{2 t-x}^{x} n(x, s) \tilde{N}(x, s+2 t) d s \\
M_{1}(x, t) & =\int_{-x}^{x-2 t} M(x, s) \tilde{N}(x, s+2 t) d s-\int_{2 t-x}^{x} M(x, s) \tilde{N}(x, s+2 t) d s \\
M_{2}(x, t) & =-\int_{-x}^{x-2 t} N(x, s) \tilde{M}(x, s+2 t) d s+\int_{2 t-x}^{x} N(x, s) \tilde{M}(x, s+2 t) d s .
\end{aligned}
$$

Let $\varphi(x, \lambda)$ and $\tilde{\varphi}(x, \lambda)$ be substituted into (1.1) and (1.7),

$$
\begin{aligned}
& -\varphi^{\prime \prime}(x, \lambda)+(2 \lambda p(x)+q(x)) \varphi(x, \lambda)=\lambda^{2} \rho(x) \varphi(x, \lambda) \\
& -\tilde{\varphi}^{\prime \prime}(x, \lambda)+(2 \lambda p(x)+q(x)) \tilde{\varphi}(x, \lambda)=\lambda^{2} \rho(x) \tilde{\varphi}(x, \lambda)
\end{aligned}
$$

The following equations are obtained using (2.5) and (2.6):

$$
\begin{aligned}
& \int_{0}^{\frac{\pi}{2}} \varphi(x, \lambda) \tilde{\varphi}(x, \lambda)[2 \lambda(p(x)-\tilde{p}(x))+(q(x)-\tilde{q}(x))] d x \\
& =\left[\tilde{\varphi}^{\prime}(x, \lambda) \varphi(x, \lambda)-\varphi^{\prime}(x, \lambda) \tilde{\varphi}(x, \lambda)\right]_{0}^{\frac{\pi}{2}}+\left.\right|_{\frac{\pi}{2}} ^{\pi}, \\
& \int_{0}^{\frac{\pi}{2}} \varphi(x, \lambda) \tilde{\varphi}(x, \lambda)[2 \lambda(p(x)-\tilde{p}(x))+(q(x)-\tilde{q}(x))] d x \\
& +\tilde{\varphi}^{\prime}(\pi, \lambda) \varphi(\pi, \lambda)-\varphi^{\prime}(\pi, \lambda) \tilde{\varphi}(\pi, \lambda)=0 .
\end{aligned}
$$

Let $Q(x)=q(x)-\tilde{q}(x)$ and $P(x)=p(x)-\tilde{p}(x)$,

$$
U(\lambda)=\int_{0}^{\frac{\pi}{2}}[2 \lambda P(x)+Q(x)] \varphi(x, \lambda) \tilde{\varphi}(x, \lambda) d x .
$$

It is obvious that the functions $\varphi(x, \lambda)$ and $\tilde{\varphi}(x, \lambda)$ are the solutions which satisfy boundary value conditions of (1.2) and (1.8), respectively, then if we consider these facts in equation (2.7), we obtain the following equation

$$
U\left(\lambda_{n}\right)=0
$$

for each eigenvalue $\lambda_{n}$. Let us marked

$$
U_{1}(\lambda)=\int_{0}^{\frac{\pi}{2}} P(x) \varphi(x, \lambda) \tilde{\varphi}(x, \lambda) d x, U_{2}(\lambda)=\int_{0}^{\frac{\pi}{2}} Q(x) \varphi(x, \lambda) \tilde{\varphi}(x, \lambda) d x .
$$

Then equations (2.7) can be rewritten as

$$
2 \lambda_{n} U_{1}\left(\lambda_{n}\right)+U_{2}\left(\lambda_{n}\right)=0 .
$$


From (2.4) and (2.7) we obtain

$$
|U(\lambda)| \leq\left(C_{1}+C_{2}|\lambda|\right) \exp (\tau \pi),
$$

where $C_{1}, C_{2}>0$ are constants for all complex $\lambda$. Since $\lambda_{n}=\tilde{\lambda}_{n}, \Delta(\lambda)=\varphi(\pi, \lambda)=$ $\tilde{\varphi}(\pi, \lambda)$, thus,

$$
U(\lambda)=\int_{0}^{\frac{\pi}{2}}[2 \lambda P(x)+Q(x)] \varphi(x, \lambda) \tilde{\varphi}(x, \lambda) d x=\Delta(\lambda)[\varphi(\pi, \lambda)-\tilde{\varphi}(\pi, \lambda)] .
$$

The function $\phi(\lambda)=\frac{U(\lambda)}{\Delta(\lambda)}$ is an entire function with respect to $\lambda$.

It follows from $\Delta(\lambda) \geq(|\lambda \beta|-C) \exp \left(\tau \xi^{+}(x)\right)$ and (2.9), $\phi(\lambda)=O(1)$ for sufficient large $|\lambda|$. We obtain $\phi(\lambda)=C$, for all $\lambda$ by Liouville's Theorem.

$$
\begin{aligned}
& \int_{0}^{\frac{\pi}{2}} \varphi(x, \lambda) \tilde{\varphi}(x, \lambda)[2 \lambda P(x)+Q(x)] d x \\
& =C\left[\left(\beta_{2}^{+}+\frac{\gamma_{2}}{2 \beta}\right) R_{1}\left(a_{2}\right) \cos \left[\lambda k^{+}(\pi)-\frac{1}{\beta} \int_{a_{2}}^{\pi} p(t) d t\right]\right. \\
& \quad+\left(\beta_{2}^{-}+\frac{\gamma_{2}}{2 \beta}\right) R_{2}\left(a_{2}\right) \cos \left[\lambda k^{-}(\pi)-\frac{1}{\beta} \int_{a_{2}}^{\pi} p(t) d t\right] \\
& \quad+\left(\beta_{2}^{-}-\frac{\gamma_{2}}{2 \beta}\right) R_{1}\left(a_{2}\right) \cos \left[\lambda s^{+}(\pi)+\frac{1}{\beta} \int_{a_{2}}^{\pi} p(t) d t\right] \\
& \left.\quad+\left(\beta_{2}^{+}-\frac{\gamma_{2}}{2 \beta}\right) R_{2}\left(a_{2}\right) \cos \left[\lambda s^{-}(\pi)+\frac{1}{\beta} \int_{a_{2}}^{\pi} p(t) d t\right]\right]+O\left(\exp \left(\tau k^{+}(\pi)\right)\right) .
\end{aligned}
$$

By the Riemann-Lebesgue lemma, for $\lambda \rightarrow \infty, \lambda \in \mathrm{R}$ we get $C=0$. Then,

$$
\begin{aligned}
2 U_{1}(\lambda)= & S^{+} \tilde{S}^{+} \int_{0}^{\frac{\pi}{2}} P(x) \cos \left(2 \lambda \xi^{+}(x)-K(x)\right) d x \\
& +S^{+} \tilde{S}^{+} \int_{0}^{\frac{\pi}{2}} P(x) \cos L(x) d x \\
& +S^{+} \tilde{S}^{-} \int_{0}^{\frac{\pi}{2}} P(x) \cos \left(2 \lambda a_{1} t-L(x)\right) d x \\
& +S^{+} \tilde{S}^{-} \int_{0}^{\frac{\pi}{2}} P(x) \cos \left(2 \lambda \alpha\left(x-a_{1}\right)-K(x)\right) d x \\
& +S^{-} \tilde{S}^{+} \int_{0}^{\frac{\pi}{2}} P(x) \cos \left(2 \lambda a_{1}+L(x)\right) d x \\
& +S^{-} \tilde{S}^{+} \int_{0}^{\frac{\pi}{2}} P(x) \cos \cos \left(2 \lambda \alpha\left(x-a_{1}\right)+K(x)\right) d x \\
& +S^{-} \tilde{S}^{-} \int_{0}^{\frac{\pi}{2}} P(x) \cos \left(2 \lambda \xi^{-}(x)+L(x)\right) d x
\end{aligned}
$$




$$
\begin{aligned}
& +S^{-} \tilde{S}^{-} \int_{0}^{\frac{\pi}{2}} P(x) \cos K(x) d x \\
& +\int_{0}^{\frac{\pi}{2}} P(x)\left(\int_{0}^{x} U_{c}(x, t) \cos (2 \lambda t-K(t)) d t\right) d x \\
& -\int_{0}^{\frac{\pi}{2}} P(x)\left(\int_{0}^{x} U_{s}(x, t) \sin (2 \lambda t-K(t)) d t\right) d x,
\end{aligned}
$$

where $\xi^{ \pm}(x)= \pm \alpha x \mp \alpha a_{1}+a_{1}, k^{ \pm}(x)=\mu^{+}\left(a_{2}\right) \pm \beta x \mp \beta a_{2}$,

$$
\begin{aligned}
s^{ \pm}(x) & =\mu^{-}\left(a_{2}\right) \pm \beta x \mp \beta a_{2}, \beta_{1}^{\mp}=\frac{1}{2}\left(\alpha_{1} \mp \frac{\beta_{1}}{\alpha}\right), \beta_{2}^{\mp}=\frac{1}{2}\left(\alpha_{2} \mp \frac{\alpha \beta_{2}}{\beta}\right) . \\
2 U_{1}(\lambda)= & \frac{S^{+}}{2} \tilde{S}^{+} \int_{0}^{\frac{\pi}{2}} P(t) e^{-i(K(t))} e^{i\left(2 \lambda \xi^{+}(t)\right)} d t+\frac{S^{+} \tilde{S}^{+}}{2} \int_{0}^{\frac{\pi}{2}} P(t) e^{i(K(t))} e^{-i\left(2 \lambda \xi^{+}(t)\right)} d t \\
& +\frac{S^{+} \tilde{S}^{-}}{2} \int_{0}^{\frac{\pi}{2}} P(t) e^{-i(L(t))} e^{i\left(2 \lambda a_{1} t\right)} d t+\frac{S^{+} \tilde{S}^{-}}{2} \int_{0}^{\frac{\pi}{2}} P(t) e^{i(L(t))} e^{-i\left(2 \lambda a_{1} t\right)} d t \\
& +\frac{S^{+} \tilde{S}^{-}}{2} \int_{0}^{\frac{\pi}{2}} P(t) e^{-i(K(t))} e^{i\left(2 \lambda \alpha\left(t-a_{1}\right)\right)} d t \\
& +\frac{S^{+} \tilde{S}^{-}}{2} \int_{0}^{\frac{\pi}{2}} P(t) e^{i(K(t))} e^{-i\left(2 \lambda \alpha\left(t-a_{1}\right)\right)} d t \\
& +\frac{S^{-} \tilde{S}^{+}}{2} \int_{0}^{\frac{\pi}{2}} P(t) e^{i(L(t))} e^{i\left(2 \lambda a_{1} t\right)} d t+\frac{S^{-} \tilde{S}^{+}}{2} \int_{0}^{\frac{\pi}{2}} P(t) e^{-i(L(t))} e^{i\left(2 \lambda a_{1} t\right)} d t \\
& +\frac{S^{-} \tilde{S}^{+}}{2} \int_{0}^{\frac{\pi}{2}} P(t) e^{i(K(t))} e^{i\left(2 \lambda \alpha\left(t-a_{1}\right)\right)} d t+\frac{S^{-} \tilde{S}^{+}}{2} \int_{0}^{\frac{\pi}{2}} P(t) e^{-i(K(t))} e^{i\left(2 \lambda \alpha\left(t-a_{1}\right)\right)} d t \\
& +\frac{S^{-} \tilde{S}^{-}}{2} \int_{0}^{\frac{\pi}{2}} P(t) e^{i(L(t))} e^{-i\left(2 \lambda \xi^{-}(t)\right)} d t+\frac{S^{-} \tilde{S}^{-}}{2} \int_{0}^{\frac{\pi}{2}} P(t) e^{-i(L(t))} e^{i\left(2 \lambda \xi^{-}(t)\right)} d t \\
& +S^{+} \tilde{S}^{+} \int_{0}^{\frac{\pi}{2}} P(x) \cos L(x) d x+S^{-} \tilde{S}^{-} \int_{0}^{\frac{\pi}{2}} P(x) \cos K(x) d x \\
& +\int_{0}^{\frac{\pi}{2}} P(x)\left(\int_{0}^{x} U_{c}(x, t) \cos (2 \lambda t-K(t)) d t\right) d x \\
& -\int_{0}^{\frac{\pi}{2}} P(x)\left(\int_{0}^{x} U_{S}(x, t) \sin (2 \lambda t-K(t)) d t\right) d x
\end{aligned}
$$

if necessary operations are performed and integrals are calculated.

$$
\begin{aligned}
2 U_{1}(\lambda) & =\frac{S^{+} \tilde{S}^{+}}{2}\left[\frac{T_{1}(\pi / 2)}{2 i \lambda \alpha} e^{i\left(2 \lambda \xi^{+}\left(\frac{\pi}{2}\right)\right)}-\frac{T_{1}(0)}{2 i \lambda \alpha} e^{2 i \lambda\left(\alpha a_{1}+a_{1}\right)}-\frac{1}{2 i \lambda \alpha} \int_{0}^{\frac{\pi}{2}} T_{1}^{\prime}(t) e^{i\left(2 \lambda \xi^{+}(t)\right)} d t\right] \\
+ & \frac{S^{+} \tilde{S}^{+}}{2}\left[-\frac{T_{2}(\pi / 2)}{2 i \lambda \alpha} e^{-i\left(2 \lambda \xi^{+}\left(\frac{\pi}{2}\right)\right)}+\frac{T_{2}(0)}{2 i \lambda \alpha} e^{-2 i \lambda\left(\alpha a_{1}+a_{1}\right)}+\frac{1}{2 i \lambda \alpha} \int_{0}^{\frac{\pi}{2}} T_{2}^{\prime}(t) e^{-i\left(2 \lambda \xi^{+}(t)\right)} d t\right] \\
+ & \frac{S^{+} \tilde{S}^{-}}{2}\left[\frac{T_{3}(\pi / 2)}{2 i \lambda \alpha} e^{i \lambda a_{1}}-\frac{T_{3}(0)}{2 i \lambda \alpha}-\frac{1}{2 i \lambda \alpha} \int_{0}^{\frac{\pi}{2}} T_{3}^{\prime}(t) e^{2 i a_{1} t} d t\right]
\end{aligned}
$$




$$
\begin{aligned}
& +\frac{S^{+} \tilde{S}^{-}}{2}\left[-\frac{T_{4}(\pi / 2)}{2 i \lambda \alpha} e^{i \lambda a_{1}}+\frac{T_{4}(0)}{2 i \lambda \alpha}+\frac{1}{2 i \lambda \alpha} \int_{0}^{\frac{\pi}{2}} T_{4}^{\prime}(t) e^{-2 i a_{1} t} d t\right] \\
& +\frac{S^{+} S^{-}}{2}\left[\frac{T_{1}(\pi / 2)}{2 i \lambda \alpha} e^{2 i \lambda \alpha\left(\frac{\pi}{2}-a_{1}\right)}-\frac{T_{1}(0)}{2 i \lambda \alpha} e^{-2 i \lambda \alpha a_{1}}-\frac{1}{2 i \lambda \alpha} \int_{0}^{\frac{\pi}{2}} T_{1}^{\prime}(t) e^{2 i \lambda \alpha\left(t-a_{1}\right)} d t\right] \\
& +\frac{S^{+} S^{-}}{2}\left[-\frac{T_{2}(\pi / 2)}{2 i \lambda \alpha} e^{-2 i \lambda \alpha\left(\frac{\pi}{2}-a_{1}\right)}+\frac{T_{2}(0)}{2 i \lambda \alpha} e^{2 i \lambda \alpha a_{1}}+\frac{1}{2 i \lambda \alpha} \int_{0}^{\frac{\pi}{2}} T_{2}^{\prime}(t) e^{-2 i \lambda \alpha\left(t-a_{1}\right)} d t\right] \\
& +\frac{S^{-} \tilde{S}^{+}}{2}\left[-\frac{T_{3}(\pi / 2)}{2 i \lambda \alpha} e^{-i \lambda a_{1} \pi}+\frac{T_{3}(0)}{2 i \lambda \alpha}+\frac{1}{2 i \lambda \alpha} \int_{0}^{\frac{\pi}{2}} T_{3}^{\prime}(t) e^{-2 i a_{1} t} d t\right] \\
& +\frac{S^{-} \tilde{S}^{+}}{2}\left[\frac{T_{4}(\pi / 2)}{2 i \lambda \alpha} e^{i \lambda a_{1} \pi}-\frac{T_{4}(0)}{2 i \lambda \alpha}-\frac{1}{2 i \lambda \alpha} \int_{0}^{\frac{\pi}{2}} T_{4}^{\prime}(t) e^{2 i a_{1} t} d t\right] \\
& +\frac{S^{-} \tilde{S}^{+}}{2}\left[-\frac{T_{1}(\pi / 2)}{2 i \lambda \alpha} e^{-2 i \lambda \alpha\left(\frac{\pi}{2}-a_{1}\right)}+\frac{T_{1}(0)}{2 i \lambda \alpha} e^{2 i \lambda \alpha a_{1}}+\frac{1}{2 i \lambda \alpha} \int_{0}^{\frac{\pi}{2}} T_{1}^{\prime}(t) e^{-2 i \lambda \alpha\left(t-a_{1}\right)} d t\right] \\
& +\frac{S^{-} \tilde{S}^{+}}{2}\left[\frac{T_{2}(\pi / 2)}{2 i \lambda \alpha} e^{2 i \lambda \alpha\left(\frac{\pi}{2}-a_{1}\right)}-\frac{T_{2}(0)}{2 i \lambda \alpha} e^{-2 i \lambda \alpha a_{1}}-\frac{1}{2 i \lambda \alpha} \int_{0}^{\frac{\pi}{2}} T_{2}^{\prime}(t) e^{2 i \lambda \alpha\left(t-a_{1}\right)} d t\right] \\
& +\frac{S^{-} \tilde{S}^{-}}{2}\left[-\frac{T_{4}(\pi / 2)}{2 i \lambda \alpha} e^{i\left(2 \lambda \xi^{-}\left(\frac{\pi}{2}\right)\right)}+\frac{T_{4}(0)}{2 i \lambda \alpha} e^{2 i \lambda\left(\alpha a_{1}+a_{1}\right)}+\frac{1}{2 i \lambda \alpha} \int_{0}^{\frac{\pi}{2}} T_{4}^{\prime}(t) e^{i\left(2 \lambda \xi^{-}(t)\right)} d t\right] \\
& +\frac{S^{-} \tilde{S}^{-}}{2}\left[\frac{T_{3}(\pi / 2)}{2 i \lambda \alpha} e^{-i\left(2 \lambda \xi^{-}\left(\frac{\pi}{2}\right)\right)}-\frac{T_{3}(0)}{2 i \lambda \alpha} e^{2 i \lambda\left(\alpha a_{1}-a_{1}\right)}-\frac{1}{2 i \lambda \alpha} \int_{0}^{\frac{\pi}{2}} T_{3}^{\prime}(t) e^{-i\left(2 \lambda \xi^{+}(t)\right)} d t\right] \\
& +\left[\frac{S^{+} \tilde{S}^{+}}{2 i} \int_{0}^{\frac{\pi}{2}} P(x) \cos L(x) d x+S^{-} \tilde{S}^{-} \int_{0}^{\frac{\pi}{2}} P(x) \cos K(x) d x\right. \\
& \left.+\frac{T_{6}(\pi / 2)}{2 i \lambda} e^{-i \pi \lambda}+\frac{T_{6}(0)}{2 i \lambda}+\frac{1}{2 i \lambda} \int_{0}^{\frac{\pi}{2}} T_{6}^{\prime}(t) e^{-2 i \lambda t} d t\right] \\
& \left.+\frac{T_{5}(0)}{2 i \lambda}-\frac{1}{2 i \lambda} \int_{0}^{\frac{\pi}{2}} T_{1}^{\prime}(t) e^{2 i \lambda t} d t\right]
\end{aligned}
$$

where

$$
\begin{gathered}
T_{1}(t)=P(t) e^{-i(K(t))}, T_{2}(t)=P(t) e^{i(K(t))}, T_{3}(t)=P(t) e^{-i(L(t))}, \\
T_{4}(t)=P(t) e^{i(L(t))}, P_{1}(t)=\int_{t}^{\frac{\pi}{2}} P(x) U_{c}(x, t) d x, P_{2}(t)=\int_{t}^{\frac{\pi}{2}} P(x) U_{s}(x, t) d x, \\
T_{5}(t)=\frac{P_{1}(t)+i P_{2}(t)}{2} e^{-i K(t)}, T_{6}(t)=\frac{P_{1}(t)-i P_{2}(t)}{2} e^{i K(t)} .
\end{gathered}
$$

By the Riemann-Lebesgue lemma $\int_{0}^{\frac{\pi}{2}} P(x) \cos L(x) d x=0, \int_{0}^{\frac{\pi}{2}} P(x) \cos K(x) d x$ $=0$ and $P\left(\frac{\pi}{2}\right)=0$ for $\lambda \rightarrow \infty$. 
Thus,

$$
\begin{aligned}
& 2 U_{1}(\lambda)=-\frac{S^{+} \tilde{S}^{+}}{4 i \lambda \alpha} \int_{0}^{\frac{\pi}{2}} T_{1}^{\prime}(t) e^{i\left(2 \lambda \xi^{+}(t)\right)} d t+\frac{S^{+} \tilde{S}^{+}}{4 i \lambda \alpha} \int_{0}^{\frac{\pi}{2}} T_{2}^{\prime}(t) e^{-i\left(2 \lambda \xi^{+}(t)\right)} d t \\
& -\frac{S^{+} \tilde{S}^{-}}{4 i \lambda \alpha} \int_{0}^{\frac{\pi}{2}} T_{3}^{\prime}(t) e^{2 i a_{1} t} d t+\frac{S^{+} \tilde{S}^{-}}{4 i \lambda \alpha} \int_{0}^{\frac{\pi}{2}} T_{4}^{\prime}(t) e^{-2 i a_{1} t} d t \\
& -\frac{S^{+} S^{-}}{4 i \lambda \alpha} \int_{0}^{\frac{\pi}{2}} T_{1}^{\prime}(t) e^{2 i \lambda \alpha\left(t-a_{1}\right)} d t+\frac{S^{+} S^{-}}{4 i \lambda \alpha} \int_{0}^{\frac{\pi}{2}} T_{2}^{\prime}(t) e^{-2 i \lambda \alpha\left(t-a_{1}\right)} d t \\
& +\frac{S^{-} \tilde{S}^{+}}{4 i \lambda \alpha} \int_{0}^{\frac{\pi}{2}} T_{3}^{\prime}(t) e^{-2 i a_{1} t} d t-\frac{S^{-} \tilde{S}^{+}}{4 i \lambda \alpha} \int_{0}^{\frac{\pi}{2}} T_{4}^{\prime}(t) e^{2 i a_{1} t} d t \\
& +\frac{S^{-} \tilde{S}^{+}}{4 i \lambda \alpha} \int_{0}^{\frac{\pi}{2}} T_{1}^{\prime}(t) e^{-2 i \lambda \alpha\left(t-a_{1}\right)} d t+\frac{S^{-} \tilde{S}^{+}}{4 i \lambda \alpha} \int_{0}^{\frac{\pi}{2}} T_{2}^{\prime}(t) e^{2 i \lambda \alpha\left(t-a_{1}\right)} d t \\
& +\frac{S^{-} \tilde{S}^{-}}{4 i \lambda \alpha} \int_{0}^{\frac{\pi}{2}} T_{4}^{\prime}(t) e^{i\left(2 \lambda \xi^{-}(t)\right)} d t-\frac{S^{-} \tilde{S}^{-}}{4 i \lambda \alpha} \int_{0}^{\frac{\pi}{2}} T_{3}^{\prime}(t) e^{-i\left(2 \lambda \xi^{-}(t)\right)} d t \\
& +\frac{i}{2 \lambda} \int_{0}^{\frac{\pi}{2}} T_{5}^{\prime}(t) e^{2 i \lambda t} d t-\frac{i}{2 \lambda} \int_{0}^{\frac{\pi}{2}} T_{6}^{\prime}(t) e^{-2 i \lambda t} d t, \\
& 2 U_{2}(\lambda)=S^{+} \tilde{S}^{+} \int_{0}^{\frac{\pi}{2}} Q(x)\left(\frac{e^{i\left(2 \lambda \xi^{+}(x)-K(x)\right)}+e^{-i\left(2 \lambda \xi^{+}(x)-K(x)\right)}}{2}\right) d x \\
& +S^{+} \tilde{S}^{-} \int_{0}^{\frac{\pi}{2}} Q(x)\left(\frac{e^{i\left(2 \lambda a_{1} t-L(x)\right)}+e^{-i\left(2 \lambda a_{1} t-L(x)\right)}}{2}\right) d x \\
& +S^{+} \tilde{S}^{-} \int_{0}^{\frac{\pi}{2}} Q(x)\left(\frac{e^{i\left(2 \lambda \alpha\left(x-a_{1}\right)-K(x)\right)}+e^{-i\left(2 \lambda \alpha\left(x-a_{1}\right)-K(x)\right)}}{2}\right) d x \\
& +S^{-} \tilde{S}^{+} \int_{0}^{\frac{\pi}{2}} Q(x)\left(\frac{e^{i\left(2 \lambda a_{1} t+L(x)\right)}+e^{-i\left(2 \lambda a_{1} t+L(x)\right)}}{2}\right) d x \\
& +S^{-} \tilde{S}^{+} \int_{0}^{\frac{\pi}{2}} Q(x)\left(\frac{e^{i\left(2 \lambda \alpha\left(x-a_{1}\right)+K(x)\right)}+e^{-i\left(2 \lambda \alpha\left(x-a_{1}\right)+K(x)\right)}}{2}\right) d x \\
& +S^{-} \tilde{S}^{-} \int_{0}^{\frac{\pi}{2}} Q(x)\left(\frac{e^{i\left(2 \lambda \xi^{-}(x)+L(x)\right)}+e^{-i\left(2 \lambda \xi^{-}(x)+L(x)\right)}}{2}\right) d x \\
& +S^{+} \tilde{S}^{+} \int_{0}^{\frac{\pi}{2}} Q(x) \cos L(x) d x+S^{-} \tilde{S}^{-} \int_{0}^{\frac{\pi}{2}} Q(x) \cos K(x) d x \\
& +\int_{0}^{\frac{\pi}{2}} Q(x)\left(\int_{0}^{x} U_{c}(x, t) \cos (2 \lambda t-K(t)) d t\right) d x \\
& -\int_{0}^{\frac{\pi}{2}} Q(x)\left(\int_{0}^{x} U_{s}(x, t) \sin (2 \lambda t-K(t)) d t\right) d x
\end{aligned}
$$


where

$$
\begin{gathered}
R_{1}(t)=Q(t) e^{-i(K(t))}, R_{2}(t)=Q(t) e^{i(K(t))}, R_{3}(t)=Q(t) e^{-i(L(t))}, \\
R_{4}(t)=Q(t) e^{i(L(t))}, Q_{1}(t)=\int_{t}^{\frac{\pi}{2}} P(x) U_{c}(x, t) d x, Q_{2}(t)=\int_{t}^{\frac{\pi}{2}} P(x) U_{s}(x, t) d x, \\
R_{5}(t)=\frac{Q_{1}(t)+i Q_{2}(t)}{2} e^{-i K(t)}, R_{6}(t)=\frac{Q_{1}(t)-i Q_{2}(t)}{2} e^{i K(t)} .
\end{gathered}
$$

By the Riemann-Lebesgue lemma $\int_{0}^{\frac{\pi}{2}} Q(x) \cos L(x) d x=0, \quad \int_{0}^{\frac{\pi}{2}} Q(x) \cos K(x) d x$ $=0$. Thus,

$$
\begin{aligned}
& 2 U_{2}(\lambda)= \frac{S^{+} \tilde{S}^{+}}{2} \int_{0}^{\frac{\pi}{2}} R_{1}(t) e^{i\left(2 \lambda \xi^{+}(t)\right)} d t+\frac{S^{+} \tilde{S}^{+}}{2} \int_{0}^{\frac{\pi}{2}} R_{2}(t) e^{-i\left(2 \lambda \xi^{+}(t)\right)} d t \\
&+\frac{S^{+} \tilde{S}^{-}}{2} \int_{0}^{\frac{\pi}{2}} R_{3}(t) e^{2 i a_{1} t} d t+\frac{S^{+} \tilde{S}^{-}}{2} \int_{0}^{\frac{\pi}{2}} R_{4}(t) e^{-2 i a_{1} t} d t \\
&+\frac{S^{+} S^{-}}{2} \int_{0}^{\frac{\pi}{2}} R_{1}(t) e^{2 i \lambda \alpha\left(t-a_{1}\right)} d t+\frac{S^{+} S^{-}}{2} \int_{0}^{\frac{\pi}{2}} R_{2}(t) e^{-2 i \lambda \alpha\left(t-a_{1}\right)} d t \\
&+\frac{S^{-} \tilde{S}^{+}}{2} \int_{0}^{\frac{\pi}{2}} R_{3}(t) e^{-2 i a_{1} t} d t+\frac{S^{-} \tilde{S}^{+}}{2} \int_{0}^{\frac{\pi}{2}} R_{4}(t) e^{2 i a_{1} t} d t \\
&+\frac{S^{-} \tilde{S}^{+}}{2} \int_{0}^{\frac{\pi}{2}} R_{1}(t) e^{-2 i \lambda \alpha\left(t-a_{1}\right)} d t+\frac{S^{-} \tilde{S}^{+}}{2} \int_{0}^{\frac{\pi}{2}} R_{2}(t) e^{2 i \lambda \alpha\left(t-a_{1}\right)} d t \\
&++\frac{S^{-} \tilde{S}^{-}}{2} \int_{0}^{\frac{\pi}{2}} R_{4}(t) e^{i\left(2 \lambda \xi^{-}(t)\right)} d t+\frac{S^{-} \tilde{S}^{-}}{2} \int_{0}^{\frac{\pi}{2}} R_{3}(t) e^{-i\left(2 \lambda \xi^{-}(t)\right)} d t \\
&+\frac{i}{2 \lambda} \int_{0}^{\frac{\pi}{2}} R_{5}(t) e^{2 i \lambda t} d t+\frac{i}{2 \lambda} \int_{0}^{\frac{\pi}{2}} R_{6}(t) e^{-2 i \lambda t} d t \\
& 2 \lambda U_{1}(\lambda)+U_{2}(\lambda)=0 .
\end{aligned}
$$

If (2.10) and (2.11) are substituted into (2.12), we get

$$
\begin{aligned}
& \frac{S^{+} \tilde{S}^{+}}{2 \alpha} \int_{0}^{\frac{\pi}{2}}\left(R_{1}(t)+i T_{1}^{\prime}(t)\right) e^{i\left(2 \lambda \xi^{+}(t)\right)} d t+\frac{S^{+} \tilde{S}^{+}}{2 \alpha} \int_{0}^{\frac{\pi}{2}}\left(R_{2}(t)-i T_{2}^{\prime}(t)\right) e^{-i\left(2 \lambda \xi^{+}(t)\right)} d t \\
& +\frac{S^{+} \tilde{S}^{-}}{2 \alpha} \int_{0}^{\frac{\pi}{2}}\left(R_{3}(t)+i T_{3}^{\prime}(t)\right) e^{2 i a_{1} t} d t+\frac{S^{+} \tilde{S}^{-}}{2 \alpha} \int_{0}^{\frac{\pi}{2}}\left(R_{4}(t)-i T_{4}^{\prime}(t)\right) e^{-2 i a_{1} t} d t \\
& +\frac{S^{+} S^{-}}{2 \alpha} \int_{0}^{\frac{\pi}{2}}\left(R_{1}(t)+i T_{1}^{\prime}(t)\right) e^{2 i \lambda \alpha\left(t-a_{1}\right)} d t+\frac{S^{+} S^{-}}{2 \alpha} \int_{0}^{\frac{\pi}{2}}\left(R_{2}(t)-i T_{2}^{\prime}(t)\right) e^{-2 i \lambda \alpha\left(t-a_{1}\right)} d t \\
& +\frac{S^{-} \tilde{S}^{+}}{2 \alpha} \int_{0}^{\frac{\pi}{2}}\left(R_{4}(t)+i T_{4}^{\prime}(t)\right) e^{-2 i a_{1} t} d t+\frac{S^{-} \tilde{S}^{+}}{2 \alpha} \int_{0}^{\frac{\pi}{2}}\left(R_{3}(t)-i T_{3}^{\prime}(t)\right) e^{2 i a_{1} t} d t \\
& +\frac{S^{-} \tilde{S}^{+}}{2} \int_{0}^{\frac{\pi}{2}}\left(R_{2}(t)+i T_{2}^{\prime}(t)\right) e^{2 i \lambda \alpha\left(t-a_{1}\right)} d t+\frac{S^{-} \tilde{S}^{+}}{2} \int_{0}^{\frac{\pi}{2}}\left(R_{1}(t)-i T_{1}^{\prime}(t)\right) e^{-2 i \lambda \alpha\left(t-a_{1}\right)} d t \\
& +\frac{S^{-} \tilde{S}^{-}}{2} \int_{0}^{\frac{\pi}{2}}\left(R_{4}(t)-i T_{4}^{\prime}(t)\right) e^{i\left(2 \lambda \xi^{-}(t)\right)} d t+\frac{S^{-} \tilde{S}^{-}}{2} \int_{0}^{\frac{\pi}{2}}\left(R_{3}(t)+i T_{3}^{\prime}(t)\right) e^{-i\left(2 \lambda \xi^{-}(t)\right)} d t
\end{aligned}
$$




$$
+\int_{0}^{\frac{\pi}{2}}\left(R_{5}(t)+i T_{5}^{\prime}(t)\right) e^{2 i \lambda t} d t+\int_{0}^{\frac{\pi}{2}}\left(R_{6}(t)-i T_{6}^{\prime}(t)\right) e^{-2 i \lambda t} d t=0 .
$$

Since the systems $\left\{e^{ \pm 2 i \lambda \xi^{+}(t)}: \lambda \in \mathrm{R}\right\},\left\{e^{ \pm 2 i \lambda a_{1} t}: \lambda \in \mathrm{R}\right\},\left\{e^{ \pm 2 i \lambda \alpha\left(t-a_{1}\right)}: \lambda \in \mathrm{R}\right\}$ and $\left\{e^{ \pm 2 i \lambda t}: \lambda \in \mathrm{R}\right\}$ are entire in $L_{2}\left(-\frac{\pi}{2}, \frac{\pi}{2}\right)$, it follows

$$
\begin{aligned}
& R_{1}(t)+i T_{1}^{\prime}(t)=0, R_{2}(t)-i T_{2}^{\prime}(t)=0, R_{3}(t)+i T_{3}^{\prime}(t)=0 \\
& R_{4}(t)-i T_{4}^{\prime}(t)=0, R_{1}(t)+i T_{1}^{\prime}(t)=0, R_{2}(t)-i T_{2}^{\prime}(t)=0 \\
& R_{4}(t)+i T_{4}^{\prime}(t)=0, R_{3}(t)-i T_{3}^{\prime}(t)=0, R_{2}(t)+i T_{2}^{\prime}(t)=0 \\
& R_{1}(t)-i T_{1}^{\prime}(t)=0, R_{4}(t)-i T_{4}^{\prime}(t)=0, R_{3}(t)+i T_{3}^{\prime}(t)=0 \\
& R_{5}(t)+i T_{5}^{\prime}(t)=0, R_{6}(t)-i T_{6}^{\prime}(t)=0 .
\end{aligned}
$$

Then, we get the following system:

$$
\begin{aligned}
& R_{5}(t)+i T_{5}^{\prime}(t)=0 \\
& R_{6}(t)-i T_{6}^{\prime}(t)=0
\end{aligned}
$$

and hence

$$
\left\{\begin{array}{l}
{\left[Q_{1}(t)+P_{1}(t) K^{\prime}(t)-P_{2}^{\prime}(t)\right]+i\left[Q_{2}(t)+P_{2}(t) K^{\prime}(t)+P_{1}^{\prime}(t)\right]=0} \\
{\left[Q_{1}(t)+P_{1}(t) K^{\prime}(t)-P_{2}^{\prime}(t)\right]-i\left[Q_{2}(t)+P_{2}(t) K^{\prime}(t)+P_{1}^{\prime}(t)\right]=0}
\end{array}\right.
$$

and

$$
\begin{gathered}
\left\{\begin{array}{c}
Q_{1}(t)+P_{1}(t) K^{\prime}(t)-P_{2}^{\prime}(t)=0 \\
Q_{2}(t)+P_{2}(t) K^{\prime}(t)+P_{1}^{\prime}(t)=0
\end{array}\right. \\
\left\{\begin{array}{c}
P^{\prime}(t)=U_{c}(t, t) P(t) \\
-\int_{t}^{\frac{\pi}{2}} U_{s}(x, t) Q(x) d x-\int_{t}^{\frac{\pi}{2}}\left(K^{\prime}(t) U_{s}(x, t)+\frac{\partial H_{s}(x, t)}{\partial t}\right) P(x) d x \\
P(t)=-\int_{t}^{\frac{\pi}{2}} P^{\prime}(x) d x \\
Q(t)=-\left(K^{\prime}(t)+U_{s}(t, t)\right) P(t) \\
-\int_{t}^{\frac{\pi}{2}} U_{c}(x, t) Q(x) d x-\int_{t}^{\frac{\pi}{2}}\left(K^{\prime}(t) U_{c}(x, t)-\frac{\partial H_{s}(x, t)}{\partial t}\right) P(x) d x .
\end{array}\right.
\end{gathered}
$$

If we mark this

$$
S(t)=\left(Q(t), P(t), P^{\prime}(t)\right)^{T}
$$

and

$$
K(x, t)=\left(\begin{array}{ccc}
U_{c}(x, t) & K^{\prime}(t) U_{c}(x, t)-\frac{\partial U_{s}(x, t)}{\partial t} & -\left(K^{\prime}(t)+U_{s}(t, t)\right) \\
0 & 0 & 1 \\
U_{s}(x, t) & K^{\prime}(t) U_{s}(x, t)+\frac{\partial U_{s}(x, t)}{\partial t} & U_{c}(x, t)
\end{array}\right)
$$

Equations (2.13) can be reduced to a vector from

$$
S(t)+\int_{t}^{\frac{\pi}{2}} K(x, t) S(x) d x=0
$$


for $0<t<\frac{\pi}{2}$.

Since equation (2.14) is a homogenous Volterra integral equation, it only has the trivial solution. Thus, we obtain $S(t)=0$ for $0<t<\frac{\pi}{2}$. This gives us $Q(t)=P(t)=0$ for $0<t<\frac{\pi}{2}$.

Thus, we obtain $q(x)=\tilde{q}(x)$ and $p(x)=\tilde{p}(x)$ on $(0, \pi)$. The proof is completed.

\section{REFERENCES}

[1] O. Acan and D. Baleanu, "A new numerical technique for solving fractional partial differential equations," Miskolc Mathematical Notes, vol. 19, no. 1, pp. 3-18, 2018.

[2] D. Alpay and I. Gohberg, "Inverse problems associated to a canonical differential system," in Recent Advances in Operator Theory and Related Topics. Birkhauser Basel, 2001, pp. 1-27.

[3] V. Ambarzumian, "Uber eine frage der eigenwerttheorie," Zeitschrift fur Physik, vol. 53, no. 9-10, pp. 690-695, sep 1929, doi: 10.1007/bf01330827.

[4] R. K. Amirov and A. A. Nabiev, "Inverse Problems for the Quadratic Pencil of the SturmLiouville Equations with Impulse," Abstract and Applied Analysis, vol. 2013, pp. 1-10, 2013, doi: 10.1155/2013/361989.

[5] R. Carlson, "An inverse spectral problem for Sturm-Liouville operators with discontinuous coefficients," Proceedings of the American Mathematical Society, vol. 120, no. 2, pp. 475-475, feb 1994, doi: 10.1090/s0002-9939-1994-1197532-5.

[6] A. Ergun and R. Amirov, "Direct and inverse problem for diffusion operator with discontinuity points," TWMS J. App. Eng. Math., vol. (9), pp. 9-21, 2019.

[7] S. Gala, Q. Liu, and M. Ragusa, "A new regularity criterion for the nematic liquid crystal flows," Applicable Analysis, vol. 91, no. 9, pp. 1741-1747, 2012.

[8] S. Gala and M. Ragusa, "Logarithmically improved regularity criterion for the boussinesq equations in besov spaces with negative indices," Applicable Analysis, vol. 95, no. 6, pp. 1271-1279, 2016.

[9] F. Gesztesy and B. Simon, "Inverse spectral analysis with partial information on the potential ii: The case of discrete spectrum," Transactions of the American Mathematical Society, vol. 352, no. 06, pp. 2765-2787, jun 2000, doi: 10.1090/s0002-9947-99-02544-1.

[10] O. H. Hald, "Discontinuous inverse eigenvalue problems," Communications on Pure and Applied Mathematics, vol. 37, no. 5, pp. 539-577, sep 1984, doi: 10.1002/cpa.3160370502.

[11] H.Hochstadt and B. Lieberman, "An Inverse Sturm-Liouville Problem with Mixed Given Data," SIAM Journal on Applied Mathematics, vol. 34, no. 4, pp. 676-680, jun 1978, doi: 10.1137/0134054.

[12] R. O. Hryniv and Y. V. Mykytyuk, "Half-inverse spectral problems for Sturm-Liouville operators with singular potentials," Inverse Problems, vol. 20, no. 5, pp. 1423-1444, jul 2004, doi: 10.1088/0266-5611/20/5/006.

[13] M. Keldysh, "On the eigenvalues and eigenfunctions of some classes of nonselfadjoint equations," Dokl. Akad. Nauk. SSSR, vol. 77, pp. 11-14, 1951.

[14] H. Koyunbakan and E. S. Panakhov, "Half-inverse problem for diffusion operators on the finite interval," Journal of Mathematical Analysis and Applications, vol. 326, no. 2, pp. 1024-1030, feb 2007, doi: 10.1016/j.jmaa.2006.03.068.

[15] B. I. Levin, Distribution of zeros of Entire Functions. American Mathematical Society, 1964, vol. 424-436.

[16] B. Levitan, Inverse Sturm-Liouville problems. Netherlands: VNU Science Press., 1987. 
[17] A. S. Markus, Introduction to the Spectral Theory of Polynomial Operator Pencils. American Mathematical Society, 2012. [Online]. Available: https://www.ebook.de/de/product/35126145/ a_s_markus_introduction_to_the_spectral_theory_of_polynomial_operator_pencils.html

[18] A. S. Ozkan, "Half-inverse Sturm-Liouville problem with boundary and discontinuity conditions dependent on the spectral parameter," Inverse Problems in Science and Engineering, vol. 22, no. 5, pp. 848-859, sep 2013, doi: 10.1080/17415977.2013.832241.

[19] L. Sakhnovich, "Half-inverse problems on the finite interval," Inverse Problems, vol. 17, no. 3, pp. 527-532, may 2001, doi: 10.1088/0266-5611/17/3/311.

[20] G. Wei and H.-K. Xu, "On the missing eigenvalue problem for an inverse sturm-liouville problem," Journal de Mathématiques Pures et Appliquées, vol. 91, no. 5, pp. 468-475, may 2009, doi: 10.1016/j.matpur.2009.01.007.

[21] C.-F. Yang, "Reconstruction of the diffusion operator from nodal data," Zeitschrift für Naturforschung A, vol. 65, no. 1-2, pp. 100-106, jan 2010, doi: 10.1515/zna-2010-1-211.

[22] C.-F. Yang and Z.-Y. Huang, "A half-inverse problem with eigenparameter dependent boundary conditions," Numerical Functional Analysis and Optimization, vol. 31, no. 6, pp. 754-762, jul 2010, doi: 10.1080/01630563.2010.490934.

[23] C.-F. Yang and X.-P. Yang, "An interior inverse problem for the Sturm-Liouville operator with discontinuous conditions," Applied Mathematics Letters, vol. 22, no. 9, pp. 1315-1319, sep 2009, doi: 10.1016/j.aml.2008.12.001.

[24] C.-F. Yang and A. Zettl, "Half Inverse Problems For Quadratic Pencils of Sturm-Liouville Operators," Taiwanese Journal of Mathematics, vol. 16, no. 5, pp. 1829-1846, sep 2012, doi: 10.11650/twjm/1500406800.

[25] V. A. Yurko, Inverse Spectral Problems for Linear Differential Operators and Their Applications. CRC Press, 2000. [Online]. Available: https://www.ebook.de/de/product/4351615/v_a_yurko_ inverse_spectral_problems_for_linear_differential_operators_and_their_applications.html

[26] R. Zhang, X.-C. Xu, C.-F. Yang, and N. Bondarenko, "Determination of the impulsive SturmLiouville operator from a set of eigenvalues," J.Inverse and III-Posed Probl, vol. 28, pp. 341-348, 2019.

\section{Author's address}

Abdullah Ergün

Cumhuriyet University, Vocational School of Sivas, Sivas, 58140, Turkey

E-mail address: aergunecumhuriyet.edu.tr 